\title{
Potential Impact of Accelerating the Primary Dose of Rotavirus Vaccine in Infants
}

\author{
Elizabeth E. Halvorson, MDa, Timothy R. Peters, MDa, Beverly M. Snively, PhD $^{\mathrm{b}}$, and \\ Katherine A. Poehling, MD, MPH ${ }^{\mathrm{a}, \mathrm{c}}$ \\ aDepartment of Pediatrics, Medical Center Boulevard, Winston-Salem, NC USA 27157 \\ bDepartment of Biostatistical Sciences, Medical Center Boulevard, Winston-Salem, NC USA \\ 27157 \\ 'Department of Epidemiology and Prevention, Wake Forest School of Medicine, Medical Center \\ Boulevard, Winston-Salem, NC USA 27157
}

\begin{abstract}
We estimated the potential impact of administering the first dose of rotavirus vaccine at 6 weeks (42 days of life) instead of 2 months of age, which is permissible for all U.S. vaccines recommended at 2 months of age, on rotavirus hospitalization rates. We used published data for hospitalization rates, vaccine coverage, and vaccine efficacy after one dose and assumed a twoweek delay in seroconversion after vaccine administration in the United States. Administering the first dose of rotavirus vaccine at 6 weeks instead of 8 weeks of age should have prevented 1110 , 1660, and 2210 rotavirus hospitalizations among U.S. infants $<3$ months of age in 2006 when the vaccine was first introduced. This estimated benefit represents a $2 \%-4 \%$ reduction in rotavirus hospitalizations among children $<5$ years of age.
\end{abstract}

\section{Keywords}

rotavirus vaccine; infants; rotavirus; hospitalizations; prevention; epidemiology

Rotavirus accounted for approximately one-third to one-half of the estimated 150,000 to 200,000 hospitalizations for gastroenteritis in children younger than 5 years old in the United States annually prior to rotavirus vaccine introduction in $2006 .{ }^{1-4}$ For each rotavirus hospitalization, the estimated median direct cost is $\$ 4565$, the average length of stay is 1.9 days, and parents lose 3.4 days of work. ${ }^{5}$ The global impact of rotavirus is even greater than that in the United States, with approximately 1400 to 1500 pediatric deaths daily or half a million pediatric deaths annually. 6,7

Two vaccines have been licensed to help prevent rotavirus infections in the United States: the pentavalent human-bovine vaccine (RotaTeq ${ }^{\circledR}$, Merck) was licensed in February 2006 and the monovalent human attenuated vaccine (Rotarix ${ }^{\circledR}$, GlaxoSmithKline) was licensed in April 2008. The Advisory Committee on Immunization Practices (ACIP) recommends

\footnotetext{
(C) 2012 Elsevier Ltd. All rights reserved.
}

Contact author: Katherine A. Poehling, M.D., M.P.H. Department of Pediatrics Wake Forest School of Medicine Medical Center Boulevard Winston-Salem, NC 27157 Telephone: 1-336-716-2540, Fax: 1-336-716-7100 kpoehlin@wakehealth.edu.

Publisher's Disclaimer: This is a PDF file of an unedited manuscript that has been accepted for publication. As a service to our customers we are providing this early version of the manuscript. The manuscript will undergo copyediting, typesetting, and review of the resulting proof before it is published in its final citable form. Please note that during the production process errors may be discovered which could affect the content, and all legal disclaimers that apply to the journal pertain. 
administration of either vaccine beginning as early as six weeks of age (42 days of life), although they are typically administered at two months of age. ${ }^{8-10}$ We sought to estimate the potential effect of accelerating the primary dose of rotavirus vaccine from eight weeks to six weeks of age on rotavirus hospitalization rates.

\section{Methods}

We used published data to estimate rotavirus hospitalization rates in young infants in the United States. These estimates are based on rotavirus surveillance studies conducted before currently licensed rotavirus vaccines were introduced in 2006 and 2008. Investigators performed capture-recapture analysis to estimate the total rotavirus disease burden in Cincinnati, Ohio. These estimates were achieved by comparison of results of an active surveillance program for enteric infections with results from concurrent passive rotavirus surveillance based on physician-ordered testing. In 1997-2002 and 2005-2006 when both active and passive surveillance were on-going, the estimated hospitalization rate per 10,000 infants $<3$ months of age with laboratory-confirmed rotavirus infection was 52 (95\% confidence interval 39-75). ${ }^{11}$ This result was consistent with a study of children hospitalized with gastroenteritis from January 1974 through July 1982, which found similar numbers of rotavirus-positive stool specimens among infants 0-3 and 4-6 months of age. ${ }^{12}$ Similarly, a retrospective stool analysis from children with diarrheal hospitalizations from 1994-2006 found that the peak age of laboratory-confirmed rotavirus hospitalizations was at 2 months of age. ${ }^{13}$ For this study, we assumed a baseline rotavirus hospitalization rate of 52 per 10,000 infants $<3$ months old.

We conservatively assumed a two-week delay in the direct immune response of an accelerated first dose of rotavirus vaccine, meaning that the benefit of vaccination at 6 weeks of age would be first seen at 8 weeks of age.

Vaccine efficacy has been documented for both the monovalent and pentavalent rotavirus vaccines. Partial series efficacy of monovalent rotavirus vaccine was 90\% (95\% CI 9-100\%) after one dose against rotavirus gastroenteritis of any severity. Partial series efficacy of pentavalent rotavirus vaccine was $82 \%$ (95\% CI 39-97\%) against severe rotavirus disease starting at 14 days post-vaccination with the first dose. ${ }^{14}$ For this analysis we conducted a sensitivity analysis using conservative estimates of $40 \%, 60 \%$, and $80 \%$ vaccine efficacy for a single dose of rotavirus vaccine.

For this analysis we assumed that $75 \%$ and $50 \%$ of infants were vaccinated, which is consistent with the 2010 national average vaccination coverage of $59 \%$ and the range by state of $42 \%$ to $82 \% .15$

To determine the projected hospitalization rate after vaccination, we used the following formula: [(Hospitalization rate per 10,000)*(Proportion not vaccinated $)]+[($ Hospitalization rate $) *($ Proportion vaccinated $) *(1-$ Vaccine efficacy as a proportion $)]=$ Projected hospitalization rate per 10,000 after vaccination

The estimated number of hospitalizations prevented equaled the difference in pre-vaccine and projected hospitalization rates multiplied by the estimated 4,251,095 live births according to the 2008 U.S. Census and by $2 / 12$ to account for the fact that vaccinating at 6 weeks instead of 8 weeks of life would impact 2 of the first 12 weeks of life. ${ }^{16} \mathrm{We}$ calculated the number of infants that needed to be vaccinated at 6 weeks (42 days of life) instead of 8 weeks of age to prevent one rotavirus hospitalization (the "number needed to treat", or NNT). This number was calculated by dividing the product of the total population of U.S. live births per the 2008 U.S. Census and the estimated proportion of infants that would be vaccinated by the estimated number of hospitalizations prevented; this NNT is 
equivalent to Kelly et al's calculation of the number needed to be vaccinated as $1 /$ (annual disease rate among the unvaccinated* vaccine effectiveness for that outcome). ${ }^{17}$

The approximate $95 \%$ confidence intervals (CI) were computed by applying these formulas to the published confidence bounds for the baseline hospitalization rate ( 39 and 75 per 10,000 infants), which assumes the number of live births, vaccination coverage and vaccine efficacy are known constants.

Statistical analyses were performed using S+ 8.1 (TIBCO Software Inc., Palo Alto, CA).

\section{Results}

We first estimated reductions in rotavirus hospitalization rates assuming that $75 \%$ of infants received the first dose of rotavirus vaccine at 6 weeks of age instead of 8 weeks, and a baseline rate of 52 rotavirus hospitalizations per 10,000 infants $<3$ months of age before introduction of currently licensed rotavirus vaccines in the United States (Table). If the single-dose vaccine efficacy is estimated to be $40 \%$, rotavirus hospitalization rates for infants with 2 additional weeks of protection in the first 12 weeks of life would be expected to drop by $5 \%$. In that case first-dose acceleration of rotavirus vaccine from 8 weeks to 6 weeks of age has the potential to prevent 1110 hospitalizations. If the single-dose vaccine efficacy is estimated to be $60 \%$, rotavirus hospitalization rates for infants would be expected to drop by $7.5 \%$, with first-dose acceleration preventing 1660 hospitalizations. If the singledose vaccine efficacy is estimated to be $80 \%$, rotavirus hospitalization rates for infants would be expected to drop by $10 \%$, with first-dose acceleration preventing 2210 hospitalizations.

We next used these same assumptions to perform number needed to treat (NNT) analyses. If the single dose vaccine efficacy is estimated to be $40 \%$, the number of infants that needed to be vaccinated at 6 weeks instead of 8 weeks of age to prevent one rotavirus hospitalization, or NNT, was 2880 (Table). For vaccine efficacy of 60\% and 80\%, the NNT was 1920 and 1440 , respectively.

The benefit of first-dose acceleration would be less if a smaller percentage of the population received the rotavirus vaccine. The estimated reductions in rotavirus hospitalization rates assuming that $50 \%$ of infants received the first dose of rotavirus vaccine at 6 weeks of age instead of 8 weeks would be lower. Because the estimated number of vaccinated infants would decrease proportionally in this scenario, the NNT remains the same.

\section{Discussion}

Vaccination is an important public health achievement that has resulted in a $99.7 \%$ reduction in childhood mortality from major infectious diseases between 1900 and $1998 .{ }^{18}$ Efforts to improve vaccine efficacy and uptake are important areas of focus as we improve childhood health. Rotavirus is a significant source of morbidity and mortality for young children worldwide. Two rotavirus vaccines were recently licensed for use in the United States and have significantly decreased disease incidence in this country. ${ }^{19,20}$ In this study, we evaluated the potential impact of accelerating administration of the first dose of rotavirus vaccine from 8 weeks to 6 weeks of age. We estimated that this change could have prevented 1110 to 2210 rotavirus hospitalizations among infants $<3$ months of life if $75 \%$ of infants were vaccinated at 6 weeks of age when rotavirus vaccine was first introduced. With the estimated direct cost of $\$ 4565$ per rotavirus hospitalization, ${ }^{5}$ preventing 1110 to 2210 rotavirus hospitalizations among U.S. infants in 2006 when rotavirus vaccine was introduced could have yielded a cost savings of approximately $\$ 5$ to $\$ 10$ million dollars. Vaccinating $75 \%$ of U.S. infants 2 weeks earlier when the vaccine was initially licensed could have 
prevented an estimated $2-4 \%$ of the estimated 60,000 rotavirus hospitalizations among children $<5$ years of age. ${ }^{4}$ To our knowledge, this is the first study to estimate the potential impact on rates of rotavirus hospitalization from first-dose acceleration of rotavirus vaccine.

Curns and colleagues have recently published estimates of the future impact of rotavirus vaccine use in the United States on rotavirus hospitalization rates. Using national hospitalization data for acute gastroenteritis, they estimated that the decrease in overall acute gastroenteritis hospitalizations from rotavirus should be detectable in 2009 for children $<1$ year age, in 2010 for children 1 year of age, and in 2011 for children $<5$ years of age. ${ }^{21}$ Our study suggests that first-dose acceleration of rotavirus vaccine would have resulted in additional decreases in rotavirus hospitalizations if implemented when the vaccine was initially licensed.

Several studies have examined the effects of accelerating the first dose of other childhood vaccines. Early administration of the diphtheria and tetanus toxoids and acellular pertussis vaccine (DTaP) at 6 weeks of age could prevent 1236 cases of pertussis, 898 hospitalizations, and 7 deaths attributable to pertussis per year in the United States. ${ }^{22}$ In Australia, accelerated administration of DTaP was estimated to prevent $9 \%$ of hospitalizations and $12 \%$ of hospital bed-days attributable to pertussis in infants. ${ }^{23}$ Study of a pertussis outbreak in Arizona resulted in the finding that the proportion of infants who completed the primary series of DTaP (along with the other routine vaccines) within the first 6 months of life was higher for those who received vaccines at the minimal dosing interval than at the standard interval. ${ }^{24}$ Similarly, early administration of the pneumococcal conjugate vaccine could prevent approximately 100 cases of invasive pneumococcal disease among young infants in the United States. ${ }^{25}$

Of note, all of the vaccines routinely given at 2 months of age can also be given at 6 weeks of age in keeping with current ACIP guidelines. Early administration of all of the scheduled primary vaccines could in fact be accomplished without extra office visits by scheduling a routine well-child check at 6 weeks instead of 2 months. ${ }^{22}$ However, it is important to emphasize that vaccination at 6 weeks of life ( 42 days of life) is acceptable but vaccination before 38 days of life is not and would result in the need for revaccination.

One concern with accelerated vaccine administration relates to the potential consequences of fever occurring in 6 week old infants as a result of rotavirus vaccination. This could lead to increased diagnostic testing or hospitalizations. While this is certainly possible, it has not been reported in the published placebo-controlled, randomized controlled trials of rotavirus vaccine performed in infants in Asia, in South Africa and Malawi, and in sub-Saharan Africa which permitted vaccination as early as 6 weeks of age. The vaccine and placebo groups for all these trials reported similar proportions with adverse events. ${ }^{26-28}$ Another theoretical concern with this approach is that parental perspectives on vaccination may differ for children at 6 weeks and 2 months of age.

This study has several limitations. Our analysis uses baseline rates of rotavirus hospitalizations in infants that were measured before 2006, and including the 1-year period after August 1998. During that period another rotavirus vaccine (RotaShield $\AA$ ) was licensed in August 1998 and administered to infants for almost 1 year before being withdrawn. ${ }^{29-31}$ The use of this prior rotavirus vaccine may have decreased estimates of rotavirus hospitalization rates used in our analysis to result in an underestimation of the potential impact of rotavirus vaccine first-dose acceleration. Our study estimates the decrease in rotavirus hospitalizations resulting from direct protection from the vaccine only. In the absence of empiric data about the vaccine efficacy of rotavirus vaccine at 6 weeks of age, we assumed the vaccine efficacy at 6 weeks of age would be the same as that reported at 8 
weeks of age. We would have underestimated the potential impact if there is both direct and indirect protection resulting from ongoing rotavirus vaccine use and increased vaccine uptake in the population. The impact of early vaccination would be greatest when a new vaccine is licensed and would decrease as exposure of young infants to rotavirus decreases. Without published rates of rotavirus hospitalizations among children $<3$ months of age since licensure of the 2006 and 2008 rotavirus vaccines, we cannot estimate the extent to which this initial benefit would decrease. Yet, many nations around the world have not yet implemented rotavirus vaccination. ${ }^{32}$ Also, if the genotypes of rotavirus change sufficiently such that rotavirus hospitalizations increase and a new rotavirus vaccine is developed, then the same theoretic benefit could occur when this new vaccine is administered but with revised estimates on the baseline hospitalization rate and vaccine efficacy. Lastly, we computed the approximate $95 \%$ confidence intervals accounting for the published confidence bounds for the baseline hospitalization rate and assuming the number of live births, vaccination coverage and vaccine efficacy are known constants. Although each confidence interval does not account for variations in vaccine efficacy or vaccine coverage, the sensitivity analyses does provide estimates in the plausible ranges of both vaccine efficacy and vaccine coverage. Additionally, this statistical approach allows others to make straightforward estimates of benefit from earlier rotavirus vaccination, using data from other countries and clinical settings.

Furthermore, this study did not examine the potential effect of acceleration of the second and third doses of rotavirus vaccine that is also permitted by current ACIP guidelines.

This study estimates the potential benefits of accelerating the first dose of rotavirus vaccine at the time of vaccine introduction. Vaccine acceleration could be achieved without an increase in the number of office visits, and similar benefits have been demonstrated for several of the other vaccinations traditionally given at 2 months of age. Therefore, a simple modification in the schedule of pediatric office visits could result in a substantial reduction in disease burden among young infants.

\section{Acknowledgments}

This project described was supported by Grant Number K23 AI065805 from the National Institute of Allergy and Infectious Diseases and by the Wachovia Research Fund. Its contents are solely the responsibility of the authors and do not necessarily represent the official views of the National Institute of Allergy and Infectious Diseases or the National Institutes of Health.

We thank Bobby and Jenny Peters and all the children that we have treated with rotavirus disease over the years who inspired this work. We also want to express our gratitude to the anonymous reviewers whose suggestions significantly enhanced this manuscript.

\section{Abbreviations}

$\begin{array}{ll}\text { ACIP } & \text { Advisory Committee on Immunization Practices } \\ \text { CI } & \text { confidence interval } \\ \text { NNT } & \text { Number Needed to Treat } \\ \text { U.S. } & \text { United States } \\ \text { VE } & \text { vaccine efficacy }\end{array}$




\section{References}

1. Curns AT, Coffin F, Glasser JW, Glass RI, Parashar UD. Projected Impact of the new rotavirus vaccination program on hospitalizations for gastroenteritis and rotavirus disease among US children $<5$ years of age during 2006-2015. J Infect Dis. 2009; 200(Suppl 1):S49-S56. [PubMed: 19817614]

2. Charles MD, Holman RC, Curns AT, Parashar UD, Glass RI, Bresee JS. Hospitalizations associated with rotavirus gastroenteritis in the United States, 1993-2002. Pediatr Infect Dis J. 2006; 25:489-93. [PubMed: 16732145]

3. Malek MA, Curns AT, Holman RC, et al. Diarrhea- and rotavirus-associated hospitalizations among children less than 5 years of age: United States, 1997 and 2000. Pediatrics. 2006; 117:1887-92. [PubMed: 16740827]

4. Fischer TK, Viboud C, Parashar U, et al. Hospitalizations and deaths from diarrhea and rotavirus among children < 5 years of age in the United States, 1993-2003. J Infect Dis. 2007; 195:1117-25. [PubMed: 17357047]

5. Mast TC, Walter EB, Bulotsky M, et al. Burden of childhood rotavirus disease on health systems in the United States. Pediatr Infect Dis J. 2010; 29:e19-e25. [PubMed: 20135751]

6. Parashar UD, Hummelman EG, Bresee JS, Miller MA, Glass RI. Global illness and deaths caused by rotavirus disease in children. Emerg Infect Dis. 2003; 9:565-72. [PubMed: 12737740]

7. Parashar UD, Burton A, Lanata C, et al. Global mortality associated with rotavirus disease among children in 2004. J Infect Dis. 2009; 200(Suppl 1):S9-S15. [PubMed: 19817620]

8. Cortese MM, Parashar UD. Prevention of rotavirus gastroenteritis among infants and children: recommendations of the Advisory Committee on Immunization Practices (ACIP). MMWR Recomm Rep. 2009; 58:1-25. [PubMed: 19194371]

9. American Academy of Pediatrics. [February 1, 2012] Bright Futures: Health Care Professionals Tools and Resources. Available at: http://brightfutures.aap.org.

10. Centers for Disease Control and Prevention. [February 1. 2012] 2012 child and adolescent immunization schedules. Available at: http://www.cdc.gov/vaccines/recs/schedules/child-schedule.htm.

11. Staat MA, Rice MA, Donauer S, et al. Estimating the rotavirus hospitalization disease burden and trends, using capture-recapture methods. Pediatr Infect Dis J. 2010; 29:1083-6. [PubMed: 21155173]

12. Brandt CD, Kim HW, Arrobio JO, et al. Epidemiology of respiratory syncytial virus infection in Washington, D.C. 3. Composite analysis of eleven consecutive yearly epidemics. Am J Epidemiol. 1973; 98:355-64. [PubMed: 4355632]

13. Clark HF, Marcello AE, Lawley D, Reilly M, DiNubile MJ. Unexpectedly high burden of rotavirus gastroenteritis in very young infants. BMC Pediatr. 2010; 10:40. [PubMed: 20540748]

14. Patel M, Shane AL, Parashar UD, Jiang B, Gentsch JR, Glass RI. Oral rotavirus vaccines: how well will they work where they are needed most? J Infect Dis. 2009; 200(Suppl 1):S39-S48. [PubMed: 19817613]

15. Tate JE, Panozzo CA, Payne DC, et al. Decline and change in seasonality of US rotavirus activity after the introduction of rotavirus vaccine. Pediatrics. 2009; 124:465-71. [PubMed: 19581260]

16. Hamilton, BE.; Martin, JA.; Ventura, SJ. [February 6, 2011] Births: Preliminary Data for 2008.; National Vital Statistics Report. p. 1-18.Available at: http://www.cdc.gov/nchs/data/nvsr/nvsr59/nvsr59_03.pdf.

17. Kelly H, Attia J, Andrews R, Heller RF. The number needed to vaccinate (NNV) and population extensions of the NNV: comparison of influenza and pneumococcal vaccine programmes for people aged 65 years and over. Vaccine. 2004; 22:2192-8. [PubMed: 15149776]

18. Guyer B, Freedman MA, Strobino DM, Sondik EJ. Annual summary of vital statistics: trends in the health of Americans during the 20th century. Pediatrics. 2000; 106:1307-17. [PubMed: 11099582]

19. Payne DC, Staat MA, Edwards KM, et al. Active, population-based surveillance for severe rotavirus gastroenteritis in children in the United States. Pediatrics. 2008; 122:1235-43. [PubMed: 19047240] 
20. Boom JA, Tate JE, Sahni LC, et al. Effectiveness of pentavalent rotavirus vaccine in a large urban population in the United States. Pediatrics. 2010; 125:e199-e207. [PubMed: 20083525]

21. Curns AT, Steiner CA, Barrett M, Hunter K, Wilson E, Parashar UD. Reduction in acute gastroenteritis hospitalizations among US children after introduction of rotavirus vaccine: analysis of hospital discharge data from 18 US states. J Infect Dis. 2010; 201:1617-24. [PubMed: 20402596]

22. Shinall MC Jr. Peters TR, Zhu Y, Chen Q, Poehling KA. Potential impact of acceleration of the pertussis vaccine primary series for infants. Pediatrics. 2008; 122:1021-6. [PubMed: 18977982]

23. Foxwell AR, McIntyre P, Quinn H, Roper K, Clements MS. Severe Pertussis in Infants: Estimated Impact of first vaccine dose at 6 versus 8 weeks in Australia. Pediatr Infect Dis J. 2011; 30:161-3. [PubMed: 20811313]

24. Bronson-Lowe D, Anderson SM. Effects of a minimum interval immunization schedule for diphtheria and tetanus toxoids and acellular pertussis vaccination during a pertussis outbreak. Arch Pediatr Adolesc Med. 2009; 163:417-21. [PubMed: 19414686]

25. Stancil JM, Peters TR, Givner LB, Poehling KA. Potential impact of accelerating the primary dose of pneumococcal conjugate vaccine in infants. Arch Pediatr Adolesc Med. 2009; 163:422-5. [PubMed: 19414687]

26. Madhi SA, Cunliffe NA, Steele D, et al. Effect of human rotavirus vaccine on severe diarrhea in African infants. N Engl J Med. 2010; 362:289-98. [PubMed: 20107214]

27. Zaman K, Dang DA, Victor JC, et al. Efficacy of pentavalent rotavirus vaccine against severe rotavirus gastroenteritis in infants in developing countries in Asia: a randomised, double-blind, placebo-controlled trial. Lancet. 2010; 376:615-23. [PubMed: 20692031]

28. Armah GE, Sow SO, Breiman RF, et al. Efficacy of pentavalent rotavirus vaccine against severe rotavirus gastroenteritis in infants in developing countries in sub-Saharan Africa: a randomised, double-blind, placebo-controlled trial. Lancet. 2010; 376:606-14. [PubMed: 20692030]

29. Rotavirus vaccine for the prevention of rotavirus gastroenteritis among children. Recommendations of the Advisory Committee on Immunization Practices (ACIP). MMWR Recomm Rep. 1999; 48:1-20.

30. Recommended childhood immunization schedule--United States, 1999. MMWR Morb Mortal Wkly Rep. 1999; 48:12-6. [PubMed: 9933123]

31. Withdrawal of rotavirus vaccine recommendation. MMWR Morb Mortal Wkly Rep. 1999; 48:1007. [PubMed: 10577495]

32. World health organization. [February 2, 2011] New and underutilized vaccine immplementation: Decision making and implementation of rotavirus vaccines. Available at: http://www.who.int/nuvi/rotavirus/decision_implementation/en/index1.html. 


\section{Highlights}

- Rotavirus vaccine can be administered at 6 weeks instead of 2 months of age.

- Administering this vaccine 2 weeks earlier should reduce rotavirus hospitalizations.

- The estimated benefit is $2-4 \%$ fewer pediatric rotavirus hospitalizations. 


\section{Table}

Projected Effect of Accelerating the First Dose of Rotavirus Vaccine from 8 Weeks to 6 Weeks of Age on Baseline of 52 Hospital Admissions Due to Rotavirus Gastroenteritis per 10,000 Infants assuming $75 \%$ or $50 \%$ of infants are vaccinated.

\begin{tabular}{|l|c|c|c|c|}
\hline $\begin{array}{c}\text { Prevaccine incidence } \\
\text { per 10,000 infants }\end{array}$ & Vaccine Efficacy & $\begin{array}{c}\text { Projected incidence per } \\
\mathbf{1 0 , 0 0 0} \text { infants }\end{array}$ & $\begin{array}{c}\text { Estimated \# of } \\
\text { Hospitalizations Prevented } \\
\mathbf{N}(\mathbf{9 5 \%} \text { CI) }\end{array}$ & $\begin{array}{c}\text { Number Needed to Treat } \\
\text { N (95\% CI) }\end{array}$ \\
\hline \multicolumn{5}{|c|}{ Assumes 75\% of infants are vaccinated } \\
\hline \multirow{3}{*}{52} & $40 \%$ & $36.4(27.3-52.5)$ & $1110(829-1590)$ & $2880(2000-3850)$ \\
\cline { 2 - 5 } & $60 \%$ & $28.6(21.5-41.3)$ & $1660(1240-2390)$ & $1920(1330-2560)$ \\
\cline { 2 - 5 } & $80 \%$ & $20.8(15.6-30.0)$ & $2210(1660-3190)$ & $1440(1000-1920)$ \\
\hline \multirow{3}{*}{52} & $40 \%$ & Assumes 50\% of infants are vaccinated & $2880(2000-3850)$ \\
\hline & $60 \%$ & $41.6(31.2-60.0)$ & $737(553-1060)$ & $1920(1330-2560)$ \\
\cline { 2 - 5 } & $80 \%$ & $36.4(27.3-52.5)$ & $1110(829-1590)$ & $1440(1000-1920)$ \\
\hline
\end{tabular}

* Number Needed to Treat is the estimated number of infants who need to be vaccinated at 6 weeks of age instead of 8 weeks of age to prevent one rotavirus hospitalization. 